\title{
A trial of intracranial pressure monitoring in traumatic brain injury
}

\author{
Samer Melhem", Lori Shutter ${ }^{2}$ and A Murat Kaynar²* \\ University of Pittsburgh Department of Critical Care Medicine: Evidence-Based Medicine Journal Club, edited by Sachin Yende
}

\section{Expanded abstract \\ Citations}

Chesnut R, Temkin N, Carney N, Dikmen S, Rondina C, Videtta W, Petroni G, Lujan S, Pridgeon J, Barber J, Machamer J, Chaddock K, Celix J, Cherner M, Hendrix T: A trial of intracranial pressure monitoring in traumatic brain injury. $N$ Engl J Med 2012, 367:2471-2481.

\section{Background}

Intracranial pressure (ICP) monitoring is considered the standard of care for severe traumatic brain injury (TBI) and is used frequently, but the efficacy of treatment based on monitoring in improving the outcome has not been rigorously assessed.

\section{Methods}

Objective: The objective was to compare efficacy of guideline-based management in which a protocol for monitoring intraparenchymal ICP was used (ICP group) or a protocol in which treatment was based on imaging and clinical examination (exam group).

Design: A multicenter randomized controlled trial was conducted.

Setting: The trial was set in ICUs in Bolivia or Ecuador.

Subjects: Patients had severe TBI $(\mathrm{n}=324)$ and were 13 years of age or older.

Interventions: Patients were randomly allocated to ICP monitoring or clinical exam-based monitoring.

Outcomes: The primary outcome was a composite of survival time, impaired consciousness, functional status at 3 and 6 months, and neuropsychological status at 6 months; neuropsychological status was assessed by an examiner who was unaware of the protocol assignment.

\footnotetext{
* Correspondence: kaynarm@upmc.edu

${ }^{2}$ Department of Critical Care Medicine, University of Pittsburgh, 3550 Terrace Street, Pittsburgh, PA 15261, USA

Full list of author information is available at the end of the article
}

This composite measure was based on performance across 21 measures of functional and cognitive status and was calculated as a percentile (with 0 indicating the worst performance, and 100 the best performance).

\section{Results}

There was no significant between-group difference in the primary outcome, a composite measure based on percentile performance across 21 measures of functional and cognitive status (score 56 in the pressure-monitoring group versus 53 in the imaging-clinical examination group; $P=0.49$ ). Six-month mortality rates were $39 \%$ in the pressuremonitoring group and $41 \%$ in the imaging-clinical examination group $(P=0.60)$. The median lengths of stay in the ICU were similar in the two groups (12 days in the pressure-monitoring group and 9 days in the imagingclinical examination group; $P=0.25$ ), although the number of days of brain-specific treatments (for example, administration of hyperosmolar fluids and the use of hyperventilation) in the ICU was higher in the imaging-clinical examination group than in the pressure-monitoring group (4.8 versus $3.4, P=0.002$ ). The distributions of serious adverse events were similar in the two groups.

\section{Conclusions}

For patients with severe TBI, care focused on maintaining monitored ICP at $20 \mathrm{mmHg}$ or less was not shown to be superior to care based on imaging and clinical examination.

\section{Commentary}

Traumatic brain injury (TBI) causes significant morbidity and mortality in the US, with 1.7 million TBIs accounting for 275,000 hospitalizations and 52,000 deaths annually [1]. At least 5.3 million Americans currently live with TBI-related disabilities, with an estimated $\$ 60.4$ billion in direct and indirect costs $[2,3]$.

Many interventions have been investigated to reduce TBI-associated sequelae. One such intervention is invasive intracranial pressure (ICP) monitoring, which is important 
as patients with ICP of greater than $20 \mathrm{mmHg}$ have worse outcomes [4]. The most recent guidelines for TBI management recommend ICP monitoring in select patients [5].

Invasive ICP monitoring is not without risks, such as infection, hemorrhage, malfunction, and additional costs. As with any monitoring in the intensive care unit, the goal is to both obtain accurate data and initiate interventions that positively affect outcomes.

Several single-center and multicenter observational studies have shown that ICP-targeted management of TBI is associated with worse outcomes, including prolonged mechanical ventilation, worse functional status, and higher risk of pneumonia, acute kidney injury, and mortality [6-8]. However, the results of these studies may be biased because they were retrospective and sicker patients may be more likely to receive ICP monitoring.

Chesnut and colleagues conducted a prospective randomized clinical trial to determine whether ICP monitoring improves outcomes. Since invasive ICP monitoring is considered standard of care, this study would have lacked clinical equipoise if conducted in the United States. Therefore, this study had to be conducted in centers that deviated from this 'standard'. The authors recruited patients from Bolivia and Ecuador, where ICP monitoring was not the standard of care. The authors concluded that ICPguided care was not superior to care based on imaging and clinical exam alone, contrary to published guidelines.

The study results suggest that management of TBI based on invasive monitoring with a goal ICP of not more than $20 \mathrm{mmHg}$ was not superior to management based on imaging and clinical exam alone, thus questioning the added risks of invasive ICP monitoring. Since this may significantly impact clinical practice patterns, a detailed assessment of the study and results must be undertaken.

Strengths of this study include its randomized, prospective nature as well as the comprehensive nature of the endpoint measured, which factored in measurements of in-hospital morbidity and quality of life after discharge.

This study has limitations. First, the authors did not report differences in pre-hospital care or care after hospital discharge. Although the study was randomized, there may have been important differences in care, including pre-hospital transport times and management and post-discharge care and rehabilitation. Unfortunately, this information is not provided in the article and may confound the results.

Second, the authors used a composite primary endpoint. They attempted to incorporate measurements of many quality-of-life issues, including mortality, cognitive impairment, and motor impairment. They produced an original, thoughtful, and complex, yet unvalidated, composite tool to measure TBI outcomes.
Third, they assessed therapeutic intensity as a secondary outcome and calculated it based on an original scoring system that bears some resemblance to the Therapeutic Intensity Level Scoring System [9] and Pediatric Intensity Level of Therapy Scoring System [10]. Based on this scale, the groups received different intensities of therapeutic interventions, with the control group receiving more treatment with mannitol, hypertonic saline, and hyperventilation $(P<0.001)$. However, the ICP group was noted to have increased barbiturate use $(P=0.02)$. What weights to assign these different interventions in a measure of treatment intensity is open to debate. Furthermore, detailed review of the protocols applied to the two groups shows small but key differences. For example, the first intervention for elevated ICP in the monitored group was to drain cerebrospinal fluid (CSF) if a ventriculostomy was in place, which was not an option in the exam group. The ICP group had 346 hours of CSF drainage, but according to a personal communication with the author, the majority of this was in a single patient who had intraventricular blood and was clearly an outlier. Other differences included the use of neuromuscular blocking agents in the ICP group and furosemide in the exam group. The results of the study would have been more robust had these differences been minimized. Finally, no information regarding the number of head computed tomography scans performed in each group was provided. Although the author stated there was not a significant difference (personal communication), this raises the question of additional radiation exposure and increased hospital costs.

An important question is whether the findings of this study can be generalized to other countries. There are differences in pre-hospital care, in-hospital management of patients with TBI, and rehabilitation services across different countries, and these differences may affect outcomes. Although invasive ICP monitoring did not affect outcomes in resource-poor settings, whether it improves outcomes in resource-rich countries is not known.

This trial is to be commended for its courage in questioning a therapy that has gained widespread acceptance as the 'standard of care' as well as its attempt to study this monitoring modality in a randomized controlled fashion. Though not providing a definitive answer as to whether ICP monitoring improves outcomes, it adds to the discussion in a thought-provoking way. ICP may represent a non-specific marker of dynamic brain pathology that draws attention to dynamic changes occurring in the brain, and both waveform analysis and cerebrovascular reactivity index can be derived from ICP monitoring, which may help guide therapy [11]. Moreover, the present study highlights the importance of clinical examinations in patient management, reminding us that reliance on monitors and measured parameters should not substitute for clinical acumen. Chesnut himself states in a commentary that 
'use of a safe and accurate quantitative index of ICP, the ICP course, and its response to treatment is much preferable to treating semi-empirically' [12]. To this end, invasive ICP monitoring is better viewed as a tool providing another piece of data for the clinician to take into account and not as an endpoint in and of itself dictating treatment.

\section{Recommendation}

There is insufficient evidence at this time to abandon treatment based on ICP monitoring, and further studies need to be done before changing practice. ICP monitoring must be used as part of a multimodal approach to the patient and viewed as an additional tool available to the clinician to manage patients with TBI.

\section{Abbreviations}

CSF: cerebrospinal fluid; ICP: intracranial pressure; TBI: traumatic brain injury.

\section{Competing interests}

The authors declare that they have no competing interests.

\section{Author details}

'Department of Anesthesiology, NYU Langone Medical Center, 550 1st Avenue, New York, NY 10016, USA. ${ }^{2}$ Department of Critical Care Medicine, University of Pittsburgh, 3550 Terrace Street, Pittsburgh, PA 15261, USA.

Published: 31 Jan 2014

\section{References}

1. Faul M, Xu L, Wald MM, Coronado VG: Traumatic Brain Injury in the United States: Emergency Department Visits, Hospitalizations, and Death. Atlanta (GA): Centers for Disease Control and Prevention, National Center for Injury Prevention and Control; 2010.
2. Thurman D, Alverson C, Dunn K, Guerrero J, Sniezek J: Traumatic brain injury in the United States: a public health perspective. J Head Trauma Rehabil 1994, 14:602-615.

3. Finkelstein E, Corso P, Miller T: The Incidence and Economic Burden of Injuries in the United States. New York (NY): Oxford University Press; 2006.

4. Narayan RK, Greenberg RP, Miller JD, Enas GG, Choi SC, Kishore PR, Selhorst JB, Lutz HA 3rd, Becker DP: Improved confidence of outcome prediction in severe head injury. J Neurosurg 1981, 54:751-762.

5. Brain Trauma Foundation; American Association of Neurological Surgeons; Congress of Neurological Surgeons: Guidelines for the management of severe traumatic brain injury. J Neurotrauma 2007, 24:S1-S106.

6. Cremer OL, van Dijk GW, van Wensen E, Brekelmans GJ, Moons KG, Leenen $L P$, Kalkman CJ: Effect of intracranial pressure monitoring and targeted intensive care on functional outcome after severe head injury. Crit Care Med 2005, 33:2207-2213.

7. Griesdale DE, McEwen J, Kurth T, Chittock DR: External ventricular drains and mortality in patients with severe traumatic brain injury. Can J Neurol Sci 2010, 37:43-48.

8. Shafi S, Diaz-Arrastia R, Madden C, Gentilello L: Intracranial pressure monitoring in brain-injured patients is associated with worsening of survival. J Trauma 2008, 64:335-340.

9. Maas Al, Harrison-Felix CL, Menon D, Adelson PD, Balkin T, Bullock R, Engel DC, Gordon W, Langlois-Orman J, Lew HL, Robertson C, Temkin N, Valadka A, Verfaellie M, Wainwright M, Wright DW, Schwab K: Standardizing data collection in traumatic brain injury. J Neurotrauma 2010, 28:177-187.

10. Shore PM, Hand LL, Roy L, Trivedi P, Kochanek PM, Adelson PD: Reliability and validity of the Pediatric Intensity Level of Therapy (PILOT) SCALE: a measure of the use of intracranial pressure-directed therapies. Crit Care Med 2006, 34:1981-1987.

11. Aries MJ, Czosnyka M, Budohoski KP, Steiner LA, Lavinio A, Kolias AG, Hutchinson PJ, Brady KM, Menon DK, Pickard JD, Smielewski P: Continuous determination of optimal cerebral perfusion pressure in traumatic brain injury. Crit Care Med 2012, 40:2456-2463.

12. Chesnut R: What the BEST TRIP study means to me as the principle investigator. [http://www.braintrauma.org/dr-chesnut-commentary]

$10.1186 / \mathrm{cc} 13713$

Cite this article as: Melhem et al:: A trial of intracranial pressure monitoring in traumatic brain injury. Critical Care 2014, 18:302 